Jean André Hammes ${ }^{1}$, Felipe Pfuetzenreiter $^{1}$, Fabrízio da Silveira ${ }^{1}$, Álvaro Koenig ${ }^{2}$, Glauco Adrieno Westphal $^{3}$

1. Médicos, Universidade da Região de Joinville - UNIVILLE - Joinville (SC), Brasil.

2. Professor de Farmacologia Clínica do Departamento de Medicina da Universidade da Região de Joinville UNIVILLE - Joinville (SC), Brasil. 3. Professor de Medicina Intensiva do Departamento de Medicina da Universidade da Região de Joinville UNIVILLE - Joinville (SC), Brasil.

Recebido da Universidade da Região de Joinville - UNIVILLE - Joinville (SC), Brasil.

Submetido em 26 de Maio de 2008 Aceito em 10 de Novembro de 2008

Autor para correspondência:

Jean André Hammes

Rua Abdon Batista, $n^{\circ}$ 744, apt 601, Ed. Presidente, Centro

CEP: 89210-010 Joinville (SC), Brasil. Fone: 047-99975967

E-mail: jeanhm@gmail.com

\section{Prevalência de potenciais interaçóes medicamentosas droga-droga em unidades de terapia intensiva}

\author{
Potential drug interactions prevalence in intensive care units
}

\section{RESUMO}

Objetivos: Interaçóes medicamentosas ocorrem quando os efeitos e/ou a toxicidade de um fármaco são alterados pela presença de outro. São geralmente imprevistas e indesejáveis. Realizado estudo com objetivo de verificar a prevalência e o valor clínico das interações medicamentosas potenciais em unidades de terapia intensiva.

Métodos: Incluídos todos pacientes de três unidades de terapia intensiva em um período de 2 meses, analisados transversalmente. Foram excluídos aqueles com tempo de permanência menor que 2 dias. Os dados foram tabulados de acordo com as combinaçóes de fármacos observadas no período de 24 horas. A presença e o valor clínico das interaçóes medicamentosas potenciais foram conferidos ao final do seguimento.

Resultados: Analisados 140 pacientes, $67,1 \%$ apresentaram alguma interaçóes medicamentosas potenciais significativa e das 1069 prescriçóes, 39,2\% tiveram este achado. De 188 interaçôes medicamentosas potenciais diferentes, 29 foram consi- deradas altamente significativas. Por análise univariada, observou-se no grupo que apresentou interação significativa maior quantidade de medicamentos, fármacos/ dia, número de médicos prescritores e tempo de internação na unidade de teapia intensiva. Por modelo de regressão logística multivariada, apenas o número de fármacos/dia correlacionou-se com o aumento do risco de interaçáo medicamentosa potencial significativa $(\mathrm{p}=0.0011)$; o uso de mais que 6 medicamentos/dia aumenta em 9.8 vezes este risco.

Conclusóes: Pacientes em unidades de terapia intensiva estáo submetidos a alto risco de interaçóes medicamentosas potenciais e o número de fármacos/dia é condição com alto valor preditivo positivo para tal. Os intensivistas devem ser alertados para o reconhecimento do problema e criados mecanismos para o manejo adequado e prudente, diminuindo iatrogenias.

Descritores: Preparaçōes farmacêuticas/efeitos adversos; Interaçóes de medicamentos; Farmacologia; Toxicidade de drogas

\section{INTRODUÇÃO}

Uma interação medicamentosa ocorre quando os efeitos e/ou a toxicidade de um fármaco são alterados pela presença de outro. ${ }^{(1,2)}$ Embora seus resultados possam ser tanto positivos (aumento da eficácia) como negativos (diminuição da eficácia, toxicidade ou idiossincrasia), elas são geralmente imprevistas e indesejáveis na farmacoterapia. ${ }^{(3)}$

Com o desenvolvimento contínuo de novos medicamentos e, conseqüentemente, prescrições com combinaçóes cada vez mais complexas, tornou-se muito difícil para médicos e farmacêuticos reconhecerem potenciais interações. ${ }^{(4)}$

$\mathrm{O}$ risco de sua ocorrência e a gravidade dependem de alguns fatores; entre os 
quais, o número de medicaçóes prescritas, duração do tratamento, idade do paciente e estados de doença. Pacientes que requerem grande número de fármacos, longo tempo de tratamento, com alteraçóes fisiológicas da idade ou certas doenças como insuficiência renal, choque, ${ }^{(5-8)}$ hepatopatias como a cirrose e hepatites virais agudas ${ }^{(9,10)}$ são considerados de alto risco para interaçóes medicamentosas severas.

Resultados do Harvard Medical Practice Study II, ${ }^{(11)}$ revelam que as complicaçóes relacionadas ao uso de medicaçóes sáo o tipo de evento adverso mais comum na internação hospitalar (19\% dos pacientes); sendo que, 2-3\% dos pacientes hospitalizados experimentam reaçóes provocadas especificamente por interaçóes farmacológicas. ${ }^{(12,13)}$ Em unidades de terapia intensiva (UTI), estudos revelam que potenciais interaçóes medicamentosas possam ocorrer em $44.3-95 \%$ dos pacientes. ${ }^{(14-16)}$ No entanto, os estudos são escassos e limitados no que se refere a medida do real valor clínico destas.

A medida deste potencial (valor clínico) deve ser viabilizada pesando a gravidade do efeito e o nível de evidência. Foi conduzido um estudo baseado em prescriçóes médicas em três UTI de Joinville (SC), com objetivo de verificar a prevalência de interaçóes medicamentosas potenciais (IMP), classificar seu valor clínico e identificar possíveis fatores de risco.

\section{MÉTODOS}

Foram identificados todos pacientes admitidos em três UTI de Joinville (SC) em dois períodos distintos: 1 de outubro a 4 de novembro de 2004 e 7 de março a 6 de abril de 2005 , tendo sido envolvidas uma UTI neuro-cirúrgica pública, uma UTI geral pública e uma UTI geral privada.

Foram incluídos no estudo todos os pacientes com tempo de permanência na UTI superior a 48 horas Foram coletados dados de prontuários e prescriçóes médicas, sendo que nas UTIs públicas a coleta foi efetuada de forma prospectiva no período acima mencionado. Posteriormente, a pesquisa foi estendida a UTI privada, onde a coleta sucedeu-se de forma retrospectiva, selecionando todos pa- cientes admitidos na UTI no mesmo período referido.

As informaçóes registradas incluíram idade, sexo, data de internação hospitalar, data de admissão na UTI, causa da admissão, escore Acute Physiological Chronic Heatlh Evaluation (APACHE) II, desfecho ao final do seguimento (alta ou óbito), prescriçóes de 24 horas e número de médicos prescritores. Foi mantida confidencialidade na coleta, pacientes e médicos não foram identificados, consentimento informado não foi considerado necessário pelas comissóes éticas das instituiçóes envolvidas.

Os dados foram tabulados de acordo com as combinações de fármacos observadas no período de 24 horas. Foram excluídas as drogas que nas prescrições manuais náo puderam ser reconhecidas por ilegibilidade, os suplementos nutricionais, os componentes hidro-eletrolíticos, insulina e vitaminas.

$\mathrm{O}$ processo de conferência de interaçóes medicamentosas potenciais foi realizada através do software $i$ Facts $^{\mathrm{TM}}$ 2005 versão para Palm OS, do mesmo autor do livro Drug Interaction Facts ${ }^{(4)}$ - sistema escolhido pela sua alta acurácia quando comparado a outros modelos. ${ }^{(17)}$

No caso da não existência do fármaco no banco de dados do $i$ Facts $^{\text {TM }}$ 2005, a combinaçâo foi considerada sem risco de potencial interação. Neste caso, não foi efetuada conferência por classe farmacológica, porque nem toda droga dentro de uma mesma classe é igualmente susceptível a interaçóes medicamentosas. ${ }^{(18)}$

Esse processo de conferência ocorreu ao final do seguimento, os pesquisadores não tiveram conhecimento das interaçóes medicamentosas potenciais durante o processo de coleta. O estudo náo contemplou meios para averiguar a real ocorrência de interaçôes.

A medida do valor clínico das IMP foi viabilizada mensurando a gravidade do efeito (intensidade) e o nível de evidência, informaçóes fornecidas pelo iFacts $^{\mathrm{TM}} 20055^{(4)}$ Quantificouse o valor clínico de 1 a 5 conforme esquema proposto pela quadro 1 que está em acordo com a literatura. ${ }^{(4,5,19)}$

Considerou-se IMP significativas aquelas com valor clínico de nível 1 a 3 sendo altamente significativas as que atendiam a condição de valor clínico 1 ou 2, correspon-

Quadro 1 - Classificação do valor clínico das interaçôes medicamentosas

\begin{tabular}{|c|c|c|c|c|c|}
\hline \multirow[b]{2}{*}{ Intensidade } & \multicolumn{5}{|c|}{ Nível de evidência } \\
\hline & Estabelecida & Provável & Suspeita & Possível & Implausível \\
\hline Grave & 1 & 1 & 1 & 4 & 5 \\
\hline Moderada & 2 & 2 & 2 & 4 & 5 \\
\hline Mínima & 3 & 3 & 3 & 5 & 5 \\
\hline
\end{tabular}

Recomendaçôes: 1 - evitar combinação; 2 - usualmente evitar combinação; 3 - minimizar o risco; 4 - nenhuma ação é requerida; 5 - não há interação; Gravidade: grave (potencial risco à vida ou dano irreversível), moderada (piora do estado clínico), mínima (leve ou imperceptível).

Fonte: (Adaptado de Jansman, 2005) (20) 
dente a intensidade grave ou moderada e evidência estabelecida ou provável.

As análises estatísticas foram efetuadas utilizando os softwares GraphPad Prism 4.0 e EPI Info 3.3. $2^{\circ}$. As diferenças estatísticas entre o grupo de pacientes que apresentou IMP de importância clínica e o grupo que não apresentou foram primeiramente avaliadas por análise univariada pelo teste não-paramétrico de Mann-Whitney - o intervalo de confiança utilizado foi de $95 \%$. As variáveis com $\mathrm{p}<0.05$ foram selecionadas para um modelo de regressão logística multivariada.

\section{RESULTADOS}

Foram analisados 140 pacientes, sendo 49 (35\%) de UTI Geral Pública, 44 (31\%) de UTI neurológica/cirúrgica pública e 47 (34\%) de UTI geral privada. A população estudada apresentou média de idade de 53,34 $\pm 20,25$, sendo 92 homens e 48 mulheres. O escore APACHE II médio foi de $18,22 \pm 7,86$. A média de drogas por dia foi de 6,76 $\pm 2,16$ com média de 13,10 $\pm 5,95$ medicamentos diferentes por paciente ao final da observação. O período analisado foi em média $10,71 \pm 12,96$ dias e o número de prescriçóes 7,64 \pm 6,66 para cada paciente.

Em relação à causa de admissão, $68(48,6 \%)$ pacientes eram cirúrgicos e $72(51,4 \%)$ clínicos. Nos primeiros, foi mais freqüente pacientes de pós-operatório de neurocirurgia (15), politrauma (13), traumatismo cranioencefálico (12) e pós-operatório de cirurgia geral (10); seguindo, pós-operatório de cirurgia cardíaca (7), pós-operatório de cirurgia torácica (4) e outras causas (7). Nos pacientes clínicos a distribuição decrescente foi insuficiência respiratória aguda (20), acidente vascular cerebral (14), septicemia (13), infarto agudo do miocárdio (6), insuficiência cardíaca (6), neoplasia (3), grande queimadura (3) e outras causas (7).
Foram avaliadas 1.069 prescriçôes de 24 horas, contendo 159 fármacos; 775 (72,5\%) apresentaram alguma IMP; 419 (39,2\%) com pelo menos uma IMP significativa, valor clínico nível 1 a 3 de Tatro (2005). De toda amostra, $123(87,9 \%)$ pacientes foram expostos a alguma IMP, $94(67,1 \%)$ com IMP significativa e $49(35 \%)$ com IMP altamente significativa. Foram detectadas 188 IMP; destas, 96 significativas e 29 altamente significativas (Tabela 1 ).

Observa-se a distribuição de acordo com o valor clínico e documentação nas figuras 1 e 2, respectivamente. Quanto ao início do efeito, $51,6 \%$ das IMP detectadas poderiam ter início tardio (após 24 horas) e 48,4\% teriam início rápido (dentro de 24 horas) caso ocorressem. Com relaçáo à gravidade, 39,7\% teriam efeitos mínimos (imperceptíveis ou leves), 50,4\% moderados (piora do estado clínico) e, 9,8\% graves (potencial risco à vida ou dano irreversível).

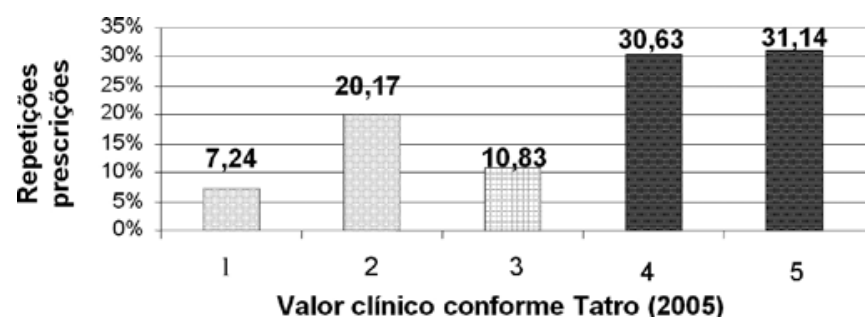

Figura 1 - Estratificação das interaçóes medicamentosas potenciais de acordo com valor clínico.

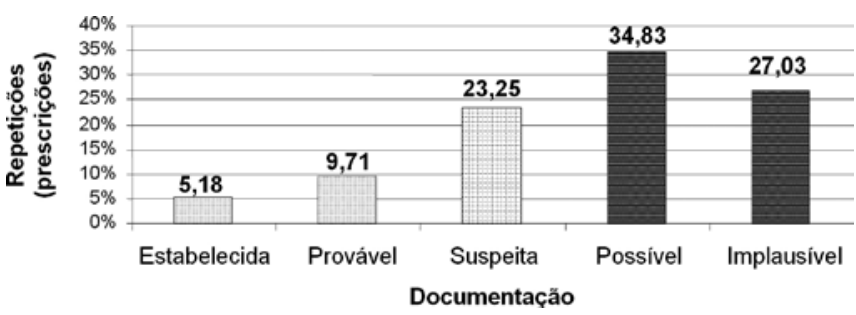

Figura 2. Estratificaçáo das interaçóes medicamentosas potenciais de acordo com nível de evidência ou documentação.

Tabela 1 - Lista das 10 interaçóes medicamentosas potenciais altamente significativas mais freqüentes

\begin{tabular}{|c|c|c|c|c|c|}
\hline Potenciais interaçóes & Valor clínico & Intensidade & Documentaçáo & Rep. & $\%(\mathrm{~N})$ \\
\hline 1 Captopril-espironolactona & 1 & Grave & Provável & 27 & $5,71(8)$ \\
\hline 2 Pancuronio - vancomicina & 2 & Moderada & Provável & 12 & $5,71(8)$ \\
\hline 3 Amicacina - piperacilina-tazobactam & 2 & Moderada & Provável & 12 & $4,29(6)$ \\
\hline 4 Digoxina - furosemida & 1 & Grave & Provável & 11 & $3,57(5)$ \\
\hline 5 AAS - hidrocortisona & 2 & Moderada & Provável & 17 & $3,57(5)$ \\
\hline 6 Dexametasona - fenitoína & 2 & Moderada & Estabelecida & 24 & $3,57(5)$ \\
\hline 7 Amiodarona - fenitoína & 2 & Moderada & Provável & 11 & $2,86(4)$ \\
\hline 8 Fenitoína - hidrocortisona & 2 & Moderada & Estabelecida & 20 & $2,86(4)$ \\
\hline 9 Amicacina - pancuronio & 1 & Grave & Provável & 7 & $2,14(3)$ \\
\hline 10 Amicacina-ampicilina-sulbactam & 2 & Moderada & Provável & 5 & $2,14(3)$ \\
\hline
\end{tabular}

Rep $=$ No de repetiçóes $=$ prescriçóes 
Entre as IMP altamente significativas detectadas, a classe farmacológica que teve maior prevalência foram os antimicrobianos (23\%) e o seu maior representante os aminoglicosídeos. A segunda classe mais prevalente foi a dos anticonvulsivantes $(10,2 \%)$, sendo a fenitoína o principal fármaco envolvido; e, os anti-hipertensivos (10,2\%), com IECA e beta-bloqueadores entre os mais presentes. A seguir, corticóides (9\%), bloqueadores neuromusculares $(7,7 \%)$, anti-arrítmicos $(6,4 \%)$ e anti-fúngicos $(5,1 \%)$. Com a prevalência no valor de 3,8\% seguem os antiagregantes plaquetários, benzodiazepínicos e diuréticos; 2,6\% anticoagulantes e broncodilatadores e 1,3\% para anestésicos, anti-eméticos, anti-psicóticos, barbitúricos, opiáceos e simpaticomiméticos.

Comparado o grupo que foi exposto a alguma IMP significativa com um grupo controle (sem IMP significativa), observou-se por análise univariada o resultado abaixo (Tabela 2).

O grupo de pacientes com IMP significativa permaneceu tempo médio de internação em dias relativamente maior $(10,73 \pm 11,96$ vs. $10,65 \pm 14,93, \mathrm{p}=0,0292)$. $\mathrm{O}$ número de fármacos foi também superior neste grupo $(14,95 \pm 5,76$ vs. $9,33 \pm 4,38, \mathrm{p}<0.0001)$; da mesma forma, o número de fármacos/dia $(7,59 \pm 1,91$ vs. $5,07 \pm$ $1,60, \mathrm{p}<0.0001)$. Não obstante, o número de profissionais prescritores envolvidos foi maior no primeiro grupo $(5,41$ $\pm 2,70$ vs. $4,37 \pm 2,79, \mathrm{p}=0,0261)$. Não houve diferenças na idade, APACHE II e tempo de internação hospitalar anterior.

Ajustado modelo de regressão logística multivariada com as variáveis que obtiveram $\mathrm{p}<0.05$ na análise univariada anterior, observou-se que apenas o número de fármacos/dia está relacionado com a presença de IMP significativa $(\mathrm{p}=0,0011)$.

Neste modelo, o uso de mais que 6 fármacos por dia aumentou em 9.8 vezes o risco de IMP significativa (sen- sibilidade $75,5 \%$, especificidade $76,1 \%$, valor preditivo positivo $86,6 \%$, valor preditivo negativo $60,3 \%$ e acurácia $75,7 \%)$.

A média de idade nos pacientes cirúrgicos foi menor que nos pacientes clínicos $(49,78 \pm 20,24$ vs. 56,71 $\pm 19,75 ; \mathrm{p}=0,0398)$. O APACHE II também foi menor nestes pacientes $(16,79 \pm 8,03$ vs. $19,57 \pm 7,39$; $\mathrm{p}$ $=0,0252)$. O número de fármacos utilizadas durante o período analisado foi maior nos pacientes clínicos (14.61 $\pm 6,07$ vs. $11,50 \pm 5,45 ; \mathrm{p}=0,0027)$, bem como a média de medicamentos por dia $(7,34 \pm 2,01$ vs. $6,15 \pm 2,17$; p $=0,0006)$. Não houve diferenças entre os dois grupos no que se refere a prescriçôes com IMP significativas $(3,22 \pm$ $3,89$ vs. $2,75 \pm 5,01 ; \mathrm{p}=$ não significativo $[\mathrm{NS}])$. $\mathrm{O}$ número de profissionais e o tempo analisado entre os grupos foi semelhante.

De acordo com a natureza da instituição, UTI pública ou privada, observou-se média de idade superior no grupo privado $(58,06 \pm 21,07$ vs. $56,71 \pm 19,80 ; p=0,0304)$. $O$ tempo de internação hospitalar precedendo admissão na UTI foi inferior na instituição privada $(5,23 \pm 14,84$ vs. $8,82 \pm 16,17, p=0,0007)$ e também o tempo de permanência na UTI $(7,60 \pm 8,61$ vs. $10,65 \pm 10,91 ; \mathrm{p}=0,0119)$. Não houveram diferenças no APACHE II, número total de medicamentos, número de fármacos/dia e número de profissionais prescritores. Não ocorreu diferença entre a prevalência de interaçóes medicamentosas potenciais.

Em relação ao grupo de pacientes sobreviventes versus não-sobreviventes, verificou-se uma maior carga de exposição diária a fármacos (medicamentos/dia) nos pacientes que evoluíram com óbito $(7,58 \pm 2,39$ vs. $6,51 \pm 2,03$; $\mathrm{p}=0,0256)$. No entanto, não houve correlaçâo com a presença de interaçóes medicamentosas significativas. Estes pacientes tinham idade mais avançada $(62,42 \pm 17,94$ vs. $50,54 \pm 20,17 ; \mathrm{p}=0,0022)$ e escore de APACHE II mais elevado (24,12 $\pm 7,48$ vs. $16,40 \pm 7,07 ; \mathrm{p}<0,0001)$.

Tabela 2 - Perfil dos pacientes de acordo com a presença ou ausência de interaçóes medicamentosas potenciais significativas

\begin{tabular}{lccc}
\hline Variáveis & Grupo com IMP significativas & Grupo sem IMP significativas & Valor de p \\
\hline Idade (anos) & $50,65 \pm 21,58(46)$ & $54,66 \pm 19,55(94)$ & NS \\
APACHE II & $17,07 \pm 8,90(46)$ & $18,79 \pm 7,29(94)$ & NS \\
Tempo de internação prévia (dias) & $7,07 \pm 13,01(46)$ & $7,28 \pm 15,36(94)$ & NS \\
Tempo na UTI (dias) & $10,65 \pm 14,93(46)$ & $10,73 \pm 11,96(94)$ & 0,0292 \\
Fármacos diferentes (N) & $9,33 \pm 4,38(46)$ & $14,95 \pm 5,76(94)$ & $<0,0001$ \\
Médicos prescritores (N) & $4,37 \pm 2,79(43)$ & $5,41 \pm 2,70(88)$ & 0,0261 \\
Prescriçốes (N) & $6,39 \pm 6,07(46)$ & $8,24 \pm 6,88(94)$ & 0,0187 \\
Fármacos por dia (N) & $5,07 \pm 1,60(46)$ & $7,59 \pm 1,91(94)$ & $<0,0001$ \\
\hline
\end{tabular}

IMP - interações medicamentosas potenciais; APACHE - Acute Physiological Chronic Health Evaluation; UTI - unidade de terapia intensiva; N - número; NS - não significativo. Resultados expressos em média \pm desvio padrão (número de pacientes analisados). Teste de Mann-Whitney 


\section{DISCUSSÃO}

O estudo demonstrou alta prevalência de IMP nas UTIs, de acordo com ensaios já realizados. ${ }^{(14-16)}$ Diferencialmente a estudos observacionais prévios, estratificou-se este risco potencial para verificar seu real valor clínico. Foi observado maior prevalência de IMP com pouco valor clínico (nível 4 e 5), para as quais nenhuma intervenção é necessária, embora número ainda importante de IMP significativas (nível 1, 2 e 3) tenha sido reconhecidas.

Levando-se em consideraçáo que os pacientes em UTI freqüentemente tem idade avançada e alteraçóes fisiológicas, somando-se condições clínicas desfavoráveis ao metabolismo dos fármacos como choque, insuficiência renal e hepatopatias, pode-se supor que a relevância das interaçóes potenciais mesmo pouco significantes tenha importância na prevenção de efeitos adversos indesejáveis.

Confirmando afirmaçóes prévias, ${ }^{(5)}$ verificou-se que os pacientes do grupo com IMP significativa utilizaram maior número de fármacos durante a internação, maior número de medicamentos/dia e maior tempo de internação na UTI - possivelmente pelo aumento da carga de exposição medicamentosa e possibilidade de combinaçóes mais complexas. Inesperadamente, o grupo de pacientes com IMP significativa também apresentou maior número de profissionais prescritores no decorrer do tratamento, fator que merece consideração.

A partir de análise multivariada, o único fator de risco independente para aumento do risco de IMP significativas foi o número de fármacos/dia, risco substancialmente aumentando quando mais que 6 variedades medicamentosas são utilizadas.

Não cabe aqui a discussão individualizada da abordagem das IMP, mas sabe-se que a maioria pode ser controlada por outros meios que não a suspensão da combinação, mas sim com ajustes de dose e monitoração dos possíveis eventos adversos, ou seja, uma avaliação individualizada de risco e benefício.

Dentre os fatores de confusão existentes nesta pesquisa, alguns não foram controlados. Os arranjos droga-droga da prescrição de 24 horas supóe que todos medicamentos estariam em uso simultâneo, mas as administrações ocorrem em horários diferentes dentro do dia e existem diferenças na velocidade de seu metabolismo. Fármacos não cadastrados no iFacts ${ }^{\text {TM }} 2005$, independente de seu par, foram consideradas como sem IMP; portanto, a prevalência de IMP pode ter sido subestimada. A pesquisa avaliou a situaçáo dos pacientes no período de estudo, muitos foram analisados em apenas um momento da sua internação; assim, nenhuma inferência confiável a respeito do tempo de internação pôde ser feita.

Existem evidências de que o risco potencial tem relação direta com a real ocorrência de interaçóes medicamentosas. Em estudo prévio envolvendo pacientes de UTI cirúrgica, verificou-se que 44,3\% dos pacientes foram expostos a IMP, 19,3\% efetivamente tiveram alteraçóes analíticas relacionadas a interação medicamentosa e 6,4\% desenvolveram manifestações clínicas. ${ }^{(14)}$ Embora o estudo tenha classificado as interaçóes em relaçáo a gravidade e documentação, a sua real ocorrência não foi contemplada na pesquisa. As IMP graves tais como captopril-espironolactona e furosemida-digoxina, drogas de associação habitual, tem ocorrência infreqüente na prática clínica. Neste contexto, novos ensaios clínicos devem ser realizados.

\section{CONCLUSÃO}

Os pacientes em UTI tem alta prevalência de interaçóes medicamentosas potenciais. O número de fármacos/dia é fator de risco independente para aumento desta possibilidade. Felizmente, a maior parte das IMP não é contraindicação ao uso do fármaco no sentido de substituição ou suspensão do uso, mas a elevada freqüência de interaçóes com valor clínico significativo (nível 1 a 3) deve ser sempre reconhecida e seus efeitos monitorados.

Sabe-se que seu reconhecimento pode ser viabilizado na prescrição, dispensação e administração das medicações, recomenda-se que seja dada maior relevância ao tema e que sistemas de apoio neste sentido devam ser habituais no exercício da terapêutica farmacológica, visando a prevenção de iatrogenias. Os sistemas de apoio a decisão baseados em evidências tem seu lugar neste âmbito e merecem maior aplicabilidade prática.

\section{ABSTRACT}

Objectives: Drug interactions occur when effects and/or toxicity of a drug are affected by presence of another drug. They are usually unpredictable and undesirable. A study was conducted to verify the prevalence and clinical value of potential drug interactions in intensive care units

Methods: All patients, of three intensive care units were included in a cross-sectional study, over a period of two months. Patients with less than a 2 days length of stay were excluded. Data were collected from twenty-four hour prescriptions and all possible paired combinations drug-drug were recorded. Prevalence and clinical value (significance) were checked at the end of follow-up.

Results: One hundred and forty patients were analyzed, 67.1\% presented with some significant potential drug interactions and of the 1069 prescriptions, $39.2 \%$ disclosed the same potential. Of 
188 different potential drug interactions, 29 were considered highly significant. Univariate analysis showed that in the group with significant potential drug interactions a higher number of different drugs, drugs/day had been used, there were more prescribing physicians and extended stay in intensive care units. Adjusted to the multivariate logistic regression model, only the number of drugs/ day correlated with increased risk of significant potential drug interaction $(\mathrm{p}=0.0011)$ and, furthermore that use of more than 6 drugs/day increased relative risk by 9.8 times.
Conclusions: Critically ill patients are submitted to high risk of potential drug interactions and the number of drugs/day has a high positive predictive value for these interactions. Therefore, it is imperative that critical care physicians be constantly alert to recognize this problem and provide appropriate mechanisms for management, thereby reducing adverse outcomes.

Keywords: Pharmaceutical preparations/adverse effects; Drug interactions; Drug toxicity; Pharmacology

\section{REFERÊNCIAS}

1. Jankel CA, Speedie SM. Detecting drug interactions: a review of the literature. DICP. 1990;24(10):982-9.

2. Hartshorn EA. Drug interactions. Fam Community Health. $1982 ; 5(2): 45-57$.

3. Streetman DS. Metabolic basis of drug interactions in the intensive care unit. Crit Care Nurs Q. 2000;22(4):1-13.

4. Tatro DS, editor. Drug interaction facts. St. Louis: Facts and Comparisons; 2005.

5. Hansten PD, Horn JR, editors. Hansten and Horn's drug interactions. St. Louis: Facts and Comparisons; 2001.

6. DiPiro JT, Hooker KD, Sherman JC, Gaines MG, Wynn JJ. Effect of experimental hemorrhagic shock on hepatic drug elimination. Crit Care Med. 1992;20(6):810-5.

7. Park GR. Molecular mechanisms of drug metabolism in the critically ill. Br J Anaesth. 1996;77(1):32-49.

8. Kennedy JM, Riji AM. Effects of surgery on the pharmacokinetic parameters of drugs. Clin Pharmacokinet. 1998;35(4):293-312.

9. Wilkinson GR, Branch RA. Effects of hepatic disease on clinical pharmacokinetics. In: Benet LZ, Massoud N, Gambertoglio JG, editors. Pharmacokinetic basis for drug treatment. New York: Raven Press; c1984.

10. Keiding S. Drug administration to liver patients: Aspects of liver pathophysiology. Semin Liver Dis. 1995;15(3):268-82.

11. Leape LL, Brennan TA, Laird N, Lawthers AG, Localio AR, Barnes BA, et al. The nature of adverse events in hospitalized patients. Results of the Harvard Medical Practice
Study II. N Engl J Med.1991;324(6):377-84.

12. Hallas J, Harvald B, Worm J, Beck-Nielsen J, Gram LF, Grodum E, et al. Drug related hospital admissions. Results from an intervention program. Eur J Clin Pharmacol. 1993;45(3):199-203.

13. Gosney M, Tallis R. Prescription of contraindicated and interacting drugs in elderly patients admitted to hospital. Lancet. 1984;2(8402):564-7.

14. Sierra P, Castillo J, Gómez M, Sorribes V, Monterde J, Castaño J. [Potential and real drug interactions in critical care patients]. Rev Esp Anestesiol Reanim. 1997;44(10):383-7. Spanish.

15. Nielsen EW, Dybwik K. [Drug interactions in an intensive care unit]. Tidsskr Nor Laegeforen. 2004;124(22):2907-8. Norwegian.

16. Meneses A, Monteiro HS. Prevalência de interaçóes medicamentosas “droga-droga” potenciais em duas UTIs (pública X privada) de Fortaleza, Brasil. Rev Bras Ter Intensiva. 2000;12(1):4-7.

17. Barrons R. Evaluation of personal digital assistant software for drug interactions. Am J Health Syst Pharm. 2004;61(4): $380-5$.

18. Herman RJ. Drug interactions and the statins. CMAJ. 1999;161(10):1281-6. Review.

19. Sjoqvist F. FASS 2000. Stockholm: LINFO Drug Information Ltd; 2000. P.1481-6.

20. Jansman FG, Jansen AJ, Coenen JL, de Graaf JC, Smith WM, Sleijter DT, Browers JR. Assessing the clinical significance of drug interactions with fluorouracil in patients with colorectal cancer. Am J Health Syst Pharm. 2005;62(17):1788-93. 